\title{
Magnetic resonance imaging, magnetisation transfer imaging, and diffusion weighted imaging correlates of optic nerve, brain, and cervical cord damage in Leber's hereditary optic neuropathy
}

\author{
M Inglese, M Rovaris, S Bianchi, L La Mantia, G L Mancardi, A Ghezzi, P Montagna, \\ F Salvi, M Filippi
}

Neuroimaging

Research Unit,

Department of

Neuroscience,

Scientific Institute

Ospedale San Raffaele,

Via Olgettina 60, 20132

Milan, Italy

$M$ Inglese

M Rovaris

M Filippi

Department of

Ophtalmology

S Bianchi

Department of

Neurology,

Neurological Institute

"Carlo Besta", Milan,

Italy

L La Mantia

Department of

Neurology, Ospedale

San Martino,

University of Genoa,

Genoa, Italy

G L Mancardi

Multiple Sclerosis

Centre, Ospedale di

Gallarate, Gallarate,

Milan, Italy

A Ghezzi

Department of

Neurological Sciences,

University of Bologna,

Bologna, Italy

P Montagna

Department of

Neurology, Ospedale

Bellaria, University of

Bologna, Bologna,

Italy

F Salvi

Correspondence to:

Dr M Filippi

filippi.massimo@hsr.it

Received 31 May 2000 and in revised form

5 September 2000

Accepted 28 September 2000

\begin{abstract}
Objectives-Leber's hereditary optic neuropathy (LHON) is a mitochondrial disease leading to bilateral loss of central vision and severe optic nerve atrophy. A subtype of LHON presents additional clinical and MRI aspects indistinguishable from those of multiple sclerosis (MS) (LHON-MS). In patients with LHON or LHON-MS, an assessment was made of $(a)$ the severity of optic nerve damage, using MRI and magnetisation transfer imaging (MTI), and (b) the presence and extent of macroscopic and microscopic pathology in the brain and cervical cord, using MRI and MT ratio (MTR) and mean diffusivity $(\bar{D})$ histogram analysis. Methods-Ten patients with LHON, four with LHON-MS, and 20 age and sex matched healthy controls were studied. For the optic nerve and the brain, dual-echo turbo spin echo (TSE), T1 weighted spin echo, and MT images were obtained. For the brain, fast fluid attenuated inversion recovery (fast FLAIR) and diffusion weighted images were also obtained. For the cervical cord, fast short tau inversion recovery (STIR) and MT images were obtained. The volume and the average MTR value of both the optic nerves were measured. MTR and $\bar{D}$ histograms of the normal appearing brain tissue (NABT) and MTR histograms of the whole cervical cord tissue were created.

Results-The mean values of optic nerve volumes and MTR were significantly lower in patients with LHON than in healthy controls. Mean NABT-MTR histogram peak height was significantly lower in patients with LHON than in controls, whereas no significant difference was found for any of the cervical cord MTR histogram derived measures. Average diffusivity $(\bar{D})$ was higher in patients with LHON than in controls. Optic nerve volume and MTR value and mean NABTMTR were lower in patients with LHON-MS than in those with LHON.

Conclusions-The severity of optic nerve pathology in LHON is measurable in vivo using MRI and MTI. MTR and $\bar{D}$ histogram analysis suggests that microscopic brain damage occurs in LHON and that it is more severe in the MS-like form of the disease. (F Neurol Neurosurg Psychiatry 2001;70:444-449)
\end{abstract}

Keywords: Leber's hereditary optic neuropathy; multiple sclerosis; magnetic resonance imaging; magnetisation transfer imaging; diffusion weighted imaging
Leber's hereditary optic neuropathy (LHON) is a mitochondrial disorder, mainly affecting young males, and displaying strict maternal inheritance in most cases. ${ }^{1-3}$ Clinically, LHON is characterised by the acute or subacute onset of loss of central vision, which is almost always bilateral, severe, and associated with optic nerve atrophy. ${ }^{45}$

In LHON, visual loss can be associated with other neurological disturbances ${ }^{6}{ }^{2}$ indicating that the damage to the CNS might be more widespread. An association between LHON and multiple sclerosis (MS) has been suggested $^{7-10}$ and evidence for such an association has been found by recent studies with MRI. ${ }^{510}$ Most of the patients with the concurrence of LHON (demonstrated by a positive family history and by the characteristic mitochondrial mutations) and neurological symptoms or signs suggestive of MS (LHONMS) have MRI abnormalities of the brain indistinguishable from those of MS. ${ }^{510}$ The same MRI pattern of abnormalities can also be found in patients with LHON with no clinical abnormalities other than the visual disturbances. ${ }^{5}$ Moreover, mitochondrial DNA mutations associated with LHON can be found in patients with MS without a family history of LHON, but in whom optic neuritis is a prominent early symptom. ${ }^{11}$

Several MRI studies of patients with LHON $^{5611-13}$ have investigated the presence of optic nerve and brain abnormalities on conventional MRI, but none of them quantified the extent and severity of optic nerve damage. In addition, although two preliminary studies using phosphorus 31 magnetic resonance spectroscopy (MRS) ${ }^{14}{ }^{15}$ indicated that the normal appearing white matter of the occipital lobe from patients with LHON and their nonaffected siblings carrying the same mitochondrial DNA mutation showed metabolic abnormalities, the presence and severity of any microscopic pathology in the brain and cervical cord of these patients have not been extensively investigated yet. All these aspects might be relevant for a better understanding of the disease, and provide useful paraclinical outcome measures to monitor its evolution.

Conventional MRI is very sensitive for the detection of abnormalities in the CNS, ${ }^{16}$ but it does not allow the quantification of tissue damage in macroscopic lesions and in the normal appearing brain tissue (NABT).${ }^{16}$ Microscopic NABT changes are known to occur in several CNS disorders, ${ }^{17}{ }^{18}$ including MS, and 
may be important in determining patients' disability. ${ }^{17}$ Magnetisation transfer imaging $(\mathrm{MTI})^{19}$ and diffusion weighted imaging $(\mathrm{DWI})^{20}$ are promising techniques for overcoming these limitations, as both of them provide quantitative indices of structural changes within and outside $\mathrm{T} 2$ visible lesions. In the present study, we obtained MRI, MTI, and DWI from patients with LHON and LHON-MS and from healthy controls. The aims of the study were: (a) to assess the severity of optic nerve damage in LHON, using MRI and MTI, and (b) to investigate the presence and extent of macroscopic and microscopic brain and cervical cord pathology in this disease, using MRI, MTI, and DWI.

\section{Materials and methods}

PATIENTS

To be included, patients had to be affected by LHON, documented by one of the three primary mitochondrial DNA mutations. ${ }^{3} \mathrm{Di}$ agnostic criteria for LHON-MS (or Harding's disease in women) were symptoms or signs of CNS dysfunction other than visual loss and the presence of multiple white matter lesions suggestive of MS on T2 weighted scans of the brain. None of the patients had to have received immunosuppressive or immunomodulating treatments during the 12 months preceding the study. A complete opthalmological evaluation was performed by one physician in all patients. Patients were also assessed neurologically by another physician and, in patients with LHON-MS, disability was scored using the expanded disability status scale (EDSS). ${ }^{21}$ Twenty age matched and sex matched healthy subjects served as controls. Their mean (SD) age was 37.3 (12.3) years. Before study entry, all subjects signed an informed consent document and the study was approved by the local ethics committee.

\section{IMAGE ACQUISITION}

Imaging was performed in a single session, using a scanner operating at 1.5 Tesla with standard head and cervical cord coils. For the optic nerve, the following sequences were obtained: (a) T2 weighted turbo spin echo (TSE) (TR/TE=4230/119, echo train length $(E T L)=15,15$ coronal slices with $3 \mathrm{~mm}$ thickness, interslice gap $=0.3 \mathrm{~mm}$, matrix size $=180 \times 512$ and field of view $(\mathrm{FOV})=156 \times 250$ $\mathrm{mm})$; (b) T1 weighted spin echo (TR/TE $=500 /$ 14, 15 coronal slices with $3 \mathrm{~mm}$ thickness, interslice gap $=0.3 \mathrm{~mm}$, matrix size $=224 \times 512$ and $\mathrm{FOV}=156 \times 250 \mathrm{~mm}$ ); (c) 2D gradient echo (GE) $\left(\mathrm{TR} / \mathrm{TE}=640 / 12\right.$, flip angle $=20^{\circ}, 15$ contiguous coronal slices with $5 \mathrm{~mm}$ thickness, matrix size $=256 \times 256$ and $F O V=250 \times 250$ $\mathrm{mm}$ ), with and without an off resonance radio frequency (RF) saturation pulse (offset frequency $=1.5 \mathrm{kHz}$, gaussian envelope duration $=16.4 \mathrm{~ms}$, flip angle $=850^{\circ}$ ). During optic nerve imaging, all subjects were asked to close their eyes and possibly avoid eye movements. For the brain, the following sequences were obtained: (a) dual echo TSE (TR=3300, $\mathrm{TE}=16 / 98, \mathrm{ETL}=5)$; (b) fast fluid attenuated inversion recovery (fast FLAIR) (TR/TE/
$\mathrm{TI}=9500 / 119 / 2200, \mathrm{ETL}=7) ;(c)$ T1 weighted spin echo (TR/TE=768/15); (d) 2D GE $\left(\mathrm{TR} / \mathrm{TE}=640 / 12\right.$, flip angle $\left.=20^{\circ}\right)$, with and without an off resonance RF saturation pulse identical to that used for optic nerve imaging; (e) pulsed gradient spin echo echo planar (interecho spacing $=0.8, \mathrm{TE}=123$ ), with diffusion gradient applied in eight non-collinear directions, chosen to cover three dimensional space uniformly. The duration and maximum amplitude for the diffusion gradients were 25 $\mathrm{ms}$ and $21 \mathrm{mTm}^{-1}$, respectively, giving a maximum $b$ factor in each direction of $1044 \mathrm{smm}^{-2}$. To optimise the measurement of diffusion, only two $b$ factors were used ( $12 \approx 0, b 2=1044 \mathrm{~s}$ $\mathrm{mm}^{-2}$ ). Fat saturation was performed using a four RF binomial pulse train to avoid chemical shift artefacts. As this sequence was not available on the scanner at the time the study was initiated, it was obtained only from 10 patients with LHON. For the dual echo, fast FLAIR, GE and T1 weighted scans, 24 contiguous axial slices were acquired with 5 $\mathrm{mm}$ thickness, $192 \times 256$ matrix and $188 \times 250$ $\mathrm{mm}$ FOV. For the DWI, 10 slices $5 \mathrm{~mm}$ thick were acquired, with the same orientation of the other scans, positioning the second last caudal slice to match exactly the central slices of the other sets. This brain portion was chosen as it is less affected by the distortions due to $\mathrm{B}_{0}$ field inhomogeneity, which can affect image coregistration. A $128 \times 128$ matrix and $250 \times 250 \mathrm{~mm}$ FOV were used. For the cervical cord, the following sequences were obtained: (a) fast short tau inversion recovery (STIR) (TR/TE/ $\mathrm{TI}=2288 / 60 / 110, \mathrm{ETL}=11$, eight sagittal slices with thickness $=3 \mathrm{~mm}$, interslice gap $=0.3 \mathrm{~mm}$, matrix size $=264 \times 512$, FOV $=280 \times 280 \mathrm{~mm}$ ); (b) $2 \mathrm{D} \mathrm{GE}\left(\mathrm{TR} / \mathrm{TE}=640 / 12\right.$, flip angle $=20^{\circ}, 20$ axial slices with thickness $=5 \mathrm{~mm}$, matrix size $=$ $256 \times 256, \mathrm{FOV}=250 \times 250 \mathrm{~mm}$ ), with and without an off resonance RF saturation pulse identical to that used for optic nerve and brain imaging.

IMAGE ANALYSIS AND POST PROCESSING Following previously published guidelines, ${ }^{22} 23$ all the scans from all subjects were reviewed consensually by two observers, unaware to whom they belonged, to identify the presence of macroscopic abnormalities. Macroscopic brain lesions were marked on the hard copies and the corresponding lesion volumes were measured by one observer, again unaware of the subjects' identity, using a semiautomated segmentation technique based on local thresholding and the marked hard copies as a reference. ${ }^{24}$

The volumes of the optic nerves were measured on $\mathrm{T} 1$ weighted images. A single observer, unaware of the subjects' identity, calculated the areas of the optic nerve sections from 11 consecutive $3 \mathrm{~mm}$ thick slices, starting from the first slice showing the chiasm backwards (towards the eye) and using a local thresholding technique for tissue segmentation. ${ }^{24}$ The optic nerve volumes were then calculated by multiplying the sum of these areas by the slice thickness. The volumes of both the optic nerves in each subject were summed and entered into the analysis. 
From the corresponding two GE images and after image coregistration using an algorithm based on mutual information, ${ }^{25}$ we derived quantitative MTR maps of the optic nerve, brain, and cervical cord, following previously described methodology. ${ }^{17}{ }^{22}{ }^{26}$ After optic nerve and chiasm segmentation from the MTR maps, which was done using the above mentioned local thresholding technique, ${ }^{24}$ the mean MTR value was calculated for the same tract considered for optic nerve volume measurements. Due to the different slice thickness, seven consecutive $5 \mathrm{~mm}$ thick MTI slices, starting from the first slice showing the chiasm backwards, were used. The MTR value for each slice was derived from a small region of interest (four pixels) placed in the centre of the optic nerve section; the pixels adjacent to the nerve borders were excluded from the analysis, to minimise the influence of partial volume effects from the CSF. For each subject, the mean MTR value between the two optic nerves was then obtained and entered into the analysis. For brain and cervical cord, MTR histogram analysis was performed. ${ }^{27}$ When brain T2 hyperintense lesions were visible on dual echo images, their mean MTR was calculated ${ }^{22}$ and the corresponding areas were excluded from the MTR histogram analysis, using a method described elsewhere. ${ }^{17}$ The final result was the creation of MTR histograms of the NABT and of the whole cervical cord tissue, respectively. Mean diffusivity $(\bar{D})$ histograms were created as previously described. ${ }^{28}$ For each histogram, the peak height, the peak location, and the average MTR and $\bar{D}$ were analyzed.

\section{STATISTICAL ANALYSIS}

MRI and MTI derived measures were distributed normally and differences in these quantities between controls and patients with LHON were assessed using the two tailed Student's $t$ test for non-paired data. To reflect the many statistical comparisons and to avoid type II errors, a significance level of $\mathrm{p} \leqslant 0.01$ was considered significant, a $\mathrm{p}$ value between 0.01 and 0.05 was considered a trend, and $\mathrm{p}$ values $>0.05$ were considered not significant. When MRI and MTI derived measures from patients with LHON-MS were considered in isolation, a formal statistical analysis was not performed, due to the relatively small sample size of these patients. The univariate correlations between MRI or MTI derived measurements and patients' clinical characteristics (age, disease duration, visual acuity) were assessed using the Spearman rank correlation coefficient, as the clinical data entering the univariate correlations were not normally distributed.

Table 1 Mean (SD) of optic nerve (ON) volumes and MTR in patients with LHON and healthy controls

\begin{tabular}{lllll}
\hline & Controls & All patients & LHON & LHON-MS \\
\hline Mean ON volume $\left(\mathrm{mm}^{3}\right)$ & $385.4(18.1)$ & $312.7(44.2)$ & $328.3(34.5)$ & $273.7(45.5)$ \\
Mean ON MTR (\%) & $36.2(2.2)$ & $30.5(3.3)$ & $30.9(2.7)$ & $29.7(4.9)$
\end{tabular}

See text for further details and statistical analysis.

\section{Results}

CLINICAL CHARACTERISTICS

Fourteen patients (10 with isolated LHON (all men) and four with LHON-MS (two men and two women)) were studied. Eleven patients had the 11778 mutation of mitochondrial DNA, two had the 3460 and one the 14 484. The mean (SD) age was $36.4(12.2)$ years (33.2 (12.3) years for LHON and 44.5 (8.5) years for LHON-MS). The median disease duration (range) was $3.0(1-26)$ years $(2.5(1-26)$ years for LHON and $16.0(2-21)$ years for LHONMS). Visual acuity was $1 / 50$ bilaterally in three patients with LHON, counting fingers on the right and 1/10 on the left in two, perception of light on the right, and hand movements on the left in one, counting fingers on the right and hand movement on the left in one, counting fingers bilaterally in two and hand movements bilaterally in one. Visual acuity was $1 / 10$ bilaterally in one patient with LHON-MS, counting fingers bilaterally in two, and hand movements bilaterally in one. All patients with isolated LHON had a neurological examination which gave normal results. Patients with LHON-MS had clinical symptoms indistinguishable from those of MS, characterised by moderate to severe paraparesis associated with mild to moderate ataxia, sensory disturbances, and urinary dysfunctions. Their median EDSS score (range) was 6.0 (3.5-7.0). Oligoclonal bands were present in CSF in three patients with LHON-MS. Two patients with LHON and two with LHON-MS had been undergoing treatment with idebenone for 1 year (180 $\mathrm{mg} /$ day orally).

\section{OPTIC NERVE}

No macroscopic T2 weighted abnormalities were visible in the optic nerve of healthy controls and patients with LHON. In table 1, the mean values (SD) of optic nerve volumes and MTR from all patients and controls are reported. The mean optic nerve volumes and MTR were significantly lower in patients with LHON than in controls $(p<0.001)$. Patients with LHON-MS had lower optic nerve volumes and MTR than those with isolated LHON. However, the differences between healthy controls and patients with LHON were still statistically significant when patients with LHON-MS were excluded from the analysis $(p<0.001$ for both optic nerve volume and MTR). There was a significant correlation between disease duration and optic nerve volume $(r=-0.7, \mathrm{p}=0.005)$.

BRAIN

No macroscopic abnormalities were seen on any of the MR scans obtained from controls and patients with isolated LHON. By definition, all the patients with LHON-MS showed multiple white matter lesions, hyperintense on dual echo and fast FLAIR images and, in part, hypointense on T1 weighted images (fig 1). Mean total lesion volumes (range) were 13.1(0.928.9) $\mathrm{ml}$ on the dual echo, $16.0(1.3-36.0) \mathrm{ml}$ on the fast FLAIR, and $3.0(0.2-6.5) \mathrm{ml}$ on the T1 weighted scans. The mean lesion MTR (range) was 33.9 (29.8-38.9)\%. 

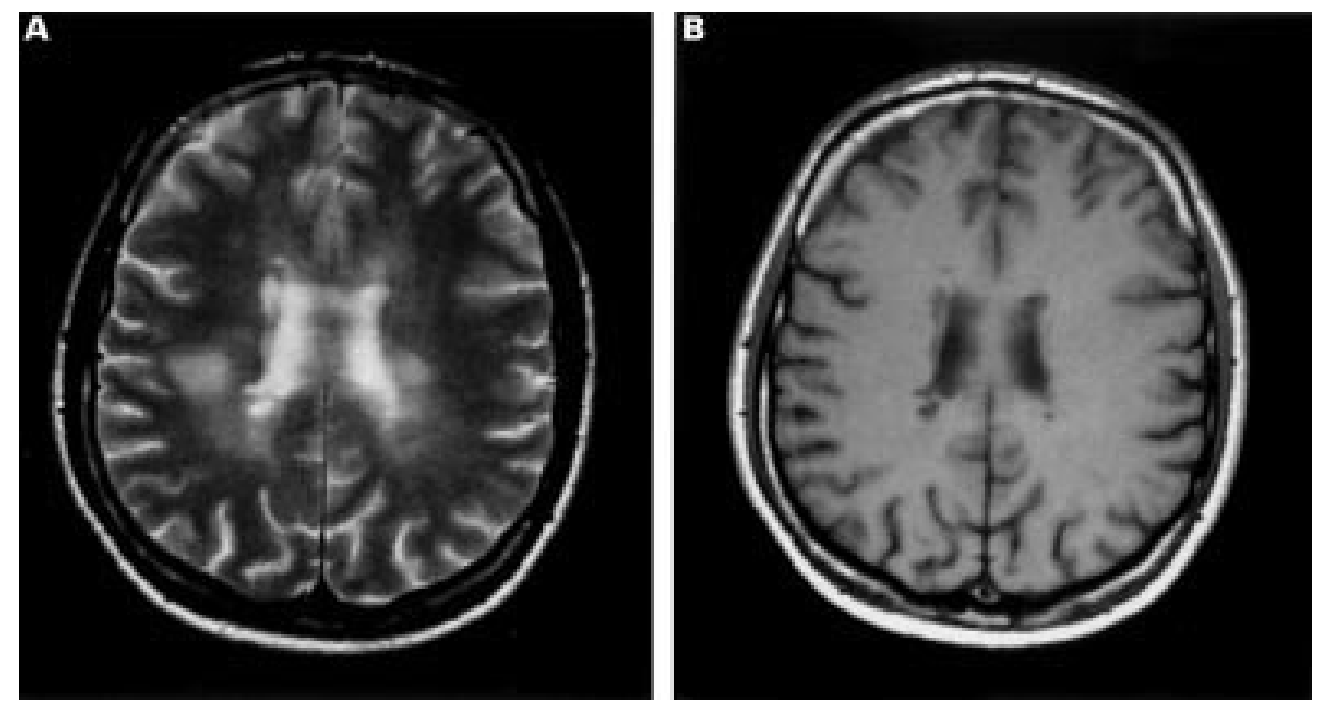

Figure 1 Axial T2 weighted turbo spin echo $(A)$ and $T 1$ weighted spin echo $(B)$ images of the brain obtained from a patient with LHON-MS at the level of the lateral ventricles. On the T2 weighted image, several hyperintense lesions are visible in the periventricular and deep white matter. On the T1 weighted image, some of these lesions appear as areas of hypointense signal. This pattern of MRI abnormalities is indistinguishable from that of MS.

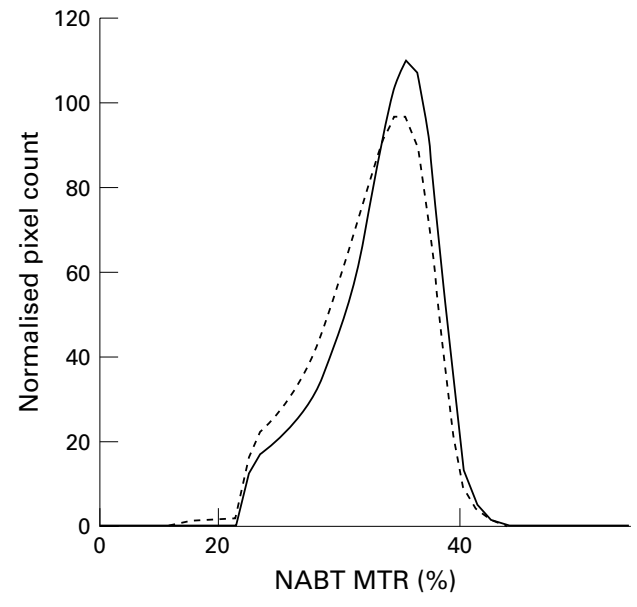

Figure 2 Normal appearing brain tissue (NABT)-MTR histograms from 20 healthy controls (full line) and 14 patients with LHON or LHON-MS (dotted line).

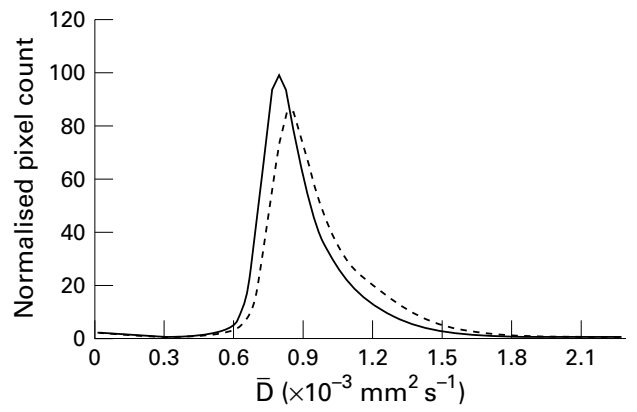

Figure $3 \quad \bar{D}$ histograms from 20 healthy controls (full line) and 10 patients with LHON (dotted line).
In table 2 , the NABT-MTR and $\bar{D}$ histogram derived measurements from all patients and controls are reported. The NABT-MTR histogram peak height was significantly lower in the group of patients as a whole than in healthy controls ( $\mathrm{p}=0.007$, fig 2). A significance trend was found for mean NABT-MTR $(p=0.02)$. There was still a significance trend for NABTMTR histogram peak height when patients with LHON-MS were excluded from the analysis $(\mathrm{p}=0.02)$. Patients with LHON had lower average $\bar{D}$ than controls $(\mathrm{p}=0.04$, fig 3$)$. NABTMTR and $\bar{D}$ histogram derived measurements from patients with LHON were also compared with the values obtained from 10 patients with MS, matched for age, sex, and disease duration. Patients with MS had significantly lower mean NABT-MTR $(p=0.01)$ and NABT-MTR histogram peak location $(\mathrm{p}=0.003)$ than patients with isolated LHON (mean values (SD) for patients with MS were $38.4(0.9) \%$ and 33.2 (1.4)\% respectively)). No significant difference or significance trend was found between patients with LHON and MS for any of the $\bar{D}$ histogram derived measurements.

\section{CERVICAL CORD}

No macroscopic abnormalities were seen on any of the scans obtained from controls and patients with LHON. One patient with LHON-MS had three lesions shorter than two vertebral segments and the remaining three patients had two lesions (fig 4); two were shorter than two vertebral segments, and one was as long as two segments. No lesions occupied the entire cord cross sectional area. None

Table 2 Mean values (SD) of NABT-MTR and D histogram derived metrics in patients with LHON and healthy controls

\begin{tabular}{lcccc}
\hline & Controls & All patients & LHON & LHON-MS \\
\hline Mean NABT-MTR (\%) & $40.0(0.5)$ & $39.4(0.8)$ & $39.6(0.8)$ & $38.9(0.5)$ \\
NABT-MTR histogram peak height & $113.1(10.5)$ & $101.7(12.7)$ & $102.1(14.3)$ & $110.5(8.6)$ \\
NABT-MTR histogram peak location (\%) & $35.3(1.0)$ & $34.8(1.0)$ & $35.2(1.0)$ & $34.0(0.8)$ \\
Average $\bar{D}\left(\times 10^{-3} \mathrm{~mm}^{2} \mathrm{~s}^{-1}\right)$ & $0.91(0.04)$ & - & $0.96(0.05)$ & - \\
$\bar{D}$ histogram peak height & $107.8(9.5)$ & - & $100.5(14.6)$ & - \\
$\bar{D}$ histogram peak location $\left(\times 10^{-3} \mathrm{~mm}^{2} \mathrm{~s}^{-1}\right)$ & $0.77(0.02)$ & - & $0.80(0.05)$ & - \\
\hline
\end{tabular}

See text for further details and statistical analysis. 


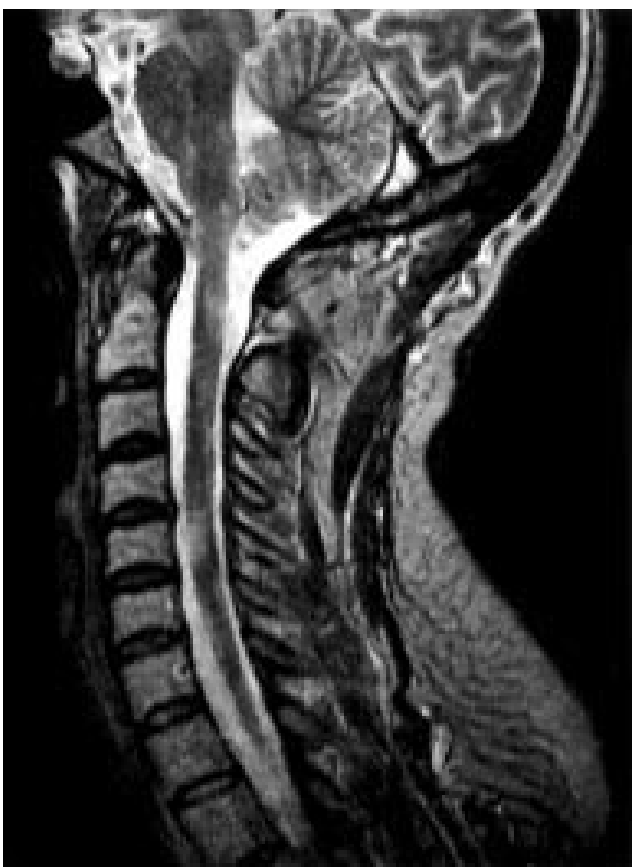

Figure 4 Sagittal STIR image of the cervical cord obtained from a patient with LHON-MS. Three

hyperintense lesions shorter than two vertebral segments are visible at different cord levels. These abnormalities are similar to those seen in patients with MS.

Table 3 Mean values (SD) of cervical cord MTR histogram derived measures in patients with LHON and healthy controls

\begin{tabular}{lll}
\hline & Controls & All patients \\
\hline Average MTR (\%) & $45.5(2.1)$ & $45.2(2.4)$ \\
Histogram peak height & $73.8(26.8)$ & $64.1(12.7)$ \\
Histogram peak location (\%) & $40.4(2.5)$ & $40.3(3.7)$ \\
\hline
\end{tabular}

See text for further details and statistical analysis.

of these lesions was associated with cord atrophy or swelling.

There were no significant differences between patients and controls for any of the cervical cord MTR histogram derived measures (table 3). These were only slightly lower in patients with LHON-MS than in patients with isolated LHON (data not shown).

\section{Discussion}

Leber's hereditary optic neuropathy is a familial disease with mitochondrial inheritance, causing severe visual loss and optic nerve atrophy. ${ }^{1-5} \mathrm{We}$ investigated the characteristics of optic nerve damage in LHON, using MRI and MTI. As previous studies reported an association between LHON and clinical ${ }^{6} \mathrm{MRI}^{5}{ }^{10}$ or MRS ${ }^{14}{ }^{15}$ findings suggesting a more widespread CNS involvement in this disease, we also assessed the presence and extent of microscopic pathology in the brain and cervical cord of patients with LHON using several MRI techniques.

We did not detect any macroscopic lesion in the optic nerve of patients with LHON. This disagrees with a previous study, ${ }^{12}$ where, using a surface eye coil, a bilateral increase of the optic nerve signal was detected on T2 weighted images in eight patients with LHON. The discrepancy between these two studies might be the reflection of the different clinical character- istics of the enrolled patient populations. In the previous study, ${ }^{12}$ patients were scanned a few months after the onset of their visual disturbances, whereas our patients, at the time they underwent the MRI examination, had a disease duration of at least 1 year. The visual assessment of optic nerve atrophy on MRI can be difficult when there are bilateral abnormalities, such as in LHON. This is confirmed by the inability of a previous MRI study ${ }^{12}$ to detect changes in optic nerve size in patients with LHON, by contrast with the high frequency of unequivocal optic atrophy reported in postmortem studies. ${ }^{45}$ Using a quantitative approach, we detected a significant reduction of the optic nerve volumes in patients with LHON compared with those of healthy controls. This is consistent with the loss of axons and myelin sheaths, which has been described in LHON. ${ }^{29}$ The correlation between disease duration and optic nerve atrophy fits well with the notion of a progressive loss of optic nerve tissue in patients with LHON.

We also obtained MT scans of the optic nerves and found that MTR values were significantly reduced in patients with LHON. A postmortem study showed that MTR values are inversely correlated with the severity of myelin and axon $\operatorname{loss}^{30}$ and several in vivo studies showed that MTR changes are correlated with patients' disability in several neurological conditions. ${ }^{1822}$ Our results confirm the potential of MTI to quantify the degree of tissue loss and indicate that this technique might also be used in the in vivo assessment of tissue damage in optic nerve diseases. Clearly, the acquisition of MT images in the optic nerve is more challenging than in the brain, because of the small size and the mobility of the optic nerves. In addition, image postprocessing is also liable to limitations related to the inclusion of pixels with partial volume effects from the CSF. Nevertheless, as shown by this and other previous studies, ${ }^{31}{ }^{32}$ it is possible to obtain reliable MTR measurements from the optic nerves. Interestingly, in patients with LHON, we did not find any significant correlation between disease duration and optic nerve MTR, by contrast with that found between disease duration and optic nerve atrophy. Although this remains speculative, one possible explanation of this discrepancy might be related to the higher sensitivity of MTI compared with optic nerve volume measurements in detecting subtle tissue changes.

No macroscopic T2 hyperintense or T1 hypointense lesions of the brain were found in patients with isolated LHON, even using fast FLAIR sequences, which are known to be more sensitive than spin echo sequences for the detection of brain white matter abnormalities. $^{33}{ }^{34}$ Consistently with other studies, ${ }^{5910}$ patients with LHON-MS showed a pattern of brain MRI abnormalities which was indistinguishable from that of patients with MS. As it is unlikely that the association between LHON and MS would represent a coincidental occurrence, given their low prevalences, this prompts speculation about the role of immunological factors in the pathogenesis of LHON and that of mitochondrial genes in 
MS. ${ }^{9-11}$ However, given the limited number of patients in our report and the conflicting evidence derived from previous research, ${ }^{5-11}$ firm conclusions cannot be drawn.

Both MTR and $\bar{D}$ histogram derived measurements were compatible with the presence of microscopic pathology in the brain of patients with LHON. In detail, patients with LHON had significantly lower NABT-MTR histogram peak height than healthy controls. This MR quantity was not significantly different from that of patients with MS. As demonstrated by previous studies in $\mathrm{MS}^{17}{ }^{22}$ and other CNS disorders, ${ }^{18}$ this finding indicates that the amount of truly normal brain tissue is reduced, due to the presence of damage not visible on T2 weighted scans. Diffusion weighted imaging provides a unique form of MRI contrast that enables the diffusional motion of water molecules to be measured in vivo ${ }^{20}$ and an increased $\bar{D}$ is the result of a net loss of barriers restricting water molecular motion. We found that the average $\bar{D}$ showed a significance trend towards increased values in patients with LHON compared with controls, but did not differ between patients with LHON and those with MS. All these findings might reflect a tissue loss and disorganisation in the optic tracts, secondary to neural fibre degeneration occurring in the retinal layer and in the prechiasmal tract of the optic nerve. However, they might also indicate that a diffuse and microscopic brain pathology is present in LHON. The second hypothesis fits with the notion of LHON as a genetic defect which should affect all the neural populations, as recently shown for other hereditary conditions, ${ }^{35}$ and raises again the issue of the pathophysiology of LHON and its relation with MS.

No macroscopic lesions were found in the cervical cord of patients with LHON, whereas all patients with LHON-MS showed a pattern of cervical cord abnormalities indistinguishable from that of MS. ${ }^{23}{ }^{36}{ }^{37}$ Cervical cord MTR histogram derived measurements were also not significantly different between patients with LHON and healthy controls. These findings suggest that the cervical cord tissue is spared in patients with LHON and fit with the hypothesis that the microscopic brain damage in these patients might be due to a secondary degeneration of retrochiasmatic optic fibres.

1 Huoponen K, Vilkki J, Aula P, et al. A new mitochondrial DNA mutation associated with Leber hereditary optic neuroretinopathy. Am f Hum Genet 1991;48:1147-53.

2 Newman NJ, Lott MT, Wallace DC. The clinical characteristic of pedigrees of Leber's hereditary optic neuropathy with the 11778 mutation. Am $\mathcal{F}$ Ophthalmol 1991;111:750-62.

3 Wallace DC, Singh G, Lott MT, et al. Mitochondrial DNA mutation associated with Leber's hereditary optic neuropathy. Science 1988;242:1427-30.

4 Johns DR, Smith KH, Miller NR. Leber's hereditary optic neuropathy. Clinical manifestations of the 3460 mutation. Arch Ophthalmol 1992;110:1577-81.

5 Riordan-Eva P, Sanders MD, Govan GG, et al. The clinical features of Leber's hereditary optic neuropathy defined by the presence of a pathogenic mitochondrial DNA mutation. Brain 1995;118:319-37.

6 Nikoskelainen EK, Marttila RJ, Huoponen K, et al. Leber's "plus": neurological abnormalities in patients with Leber's hereditary optic neuropathy. F Neurol Neurosurg Psychiatry 1995;59:160-4

7 Flanigan KM, Johns DR. Association of the 11778 mutation and demyelinating disease. Neurology 1993;43:2720-2.

8 Olsen NK, Hansen AW, Nørby S, et al. Leber's hereditary optic neuropathy associated with a disorder indistinguishable from multiple sclerosis in a male harbouring the mitochondrial DNA 11778 mutation. Acta Neurol Scand 1995; 91:326-9.
9 Harding AE, Sweeney MG, Miller DH, et al. Occurrence of a multiple sclerosis-like illness in women who have a Leber's hereditary optic neuropathy mitochondrial DNA mutation. Brain 1992:115:979-89.

10 Horvath R, Abicht A, Shoubridge EA, et al. Leber's herediary optic neuropathy presenting as multiple sclerosis-like disease of the CNS. F Neurol 2000;247:65-7.

11 Kellar-Wood H, Robertson N, Govan GG, et al. Leber's hereditary optic neuropathy mitochondrial DNA mutations in multiple sclerosis. Ann Neurol 1994;36:109-12.

12 Kermode AG, Moseley IF, Kendall BE, et al. Magnetic resonance imaging in Leber's optic neuropathy. 7 Neurol Neurosurg Psychiatry 1989;52:671-4.

13 Morrissey SP, Borruat FX, Miller DH, et al. Bilateral simultaneous optic neuropathy in adults: clinical, imaging, serological, and genetic studies. F Neurol Neurosurg Psychiatry 1995;58:70-4.

14 Cortelli P, Montagna P, Avoni P, et al. Leber's hereditary optic neuropathy: genetic, biochemical and phosphorus magnetic resonance spectroscopy study in an Italian family. Neurology 1991;41:1211-15.

15 Barbiroli B, Montagna P, Cortelli P, et al. Defective brain and muscle energy metabolism shown by in vivo ${ }^{31} \mathrm{P}$ magnetic resonance spectroscopy in nonaffected carriers of magnetic resonance spectroscopy in nonaffected carrie

16 Rovaris M, Filippi M. Magnetic resonance techniques to monitor disease evolution and treatment trial outcomes in multiple sclerosis. Curr Opin Neurol 1999;12:337-44.

17 Tortorella C, Viti B, Bozzali M, et al. A magnetization transfer histogram study of normal appearing brain tissue in multiple sclerosis. Neurology 2000;54:186-93.

18 Rovaris M, Viti B, Ciboddo G, et al. Brain involvement in systemic immune-mediated diseases: magnetic resonance and magnetisation transfer imaging study. $f$ Neurol Neurosurg Psychiatry 2000;68:170-7.

19 McGowan JC, Filippi M, Campi A, et al. Magnetisation transfer imaging: theory and application to multiple sclerosis. F Neurol Neurosurg Psychiatry 1998;64(suppl 1): S66-9.

20 Le Bihan D. Separation of diffusion and perfusion in intravoxel inchoerent motion (IVIM) MR imaging. Radiology 1988;168:497-505.

21 Kurtzke JF. Rating neurological impairment in multiple sclerosis: an expanded disability status scale (EDSS). Neurology 1983;33:1444-52.

22 Filippi M, Iannucci G, Tortorella C, et al. Comparison of MS clinical phenotypes using conventional and magnetization transfer MRI. Neurology 1999;52:588-94.

23 Rocca MA, Mastronardo G, Horsfield MA, et al. Comparison of three MR sequences for the detection of cervical cord lesions in multiple sclerosis. AfNR Am $\mathcal{F}$ Neuroradiol 1999;20:1710-16.

24 Rovaris M, Filippi M, Calori G, et al. Intra-observer reproducibility in measuring new putative markers of demyelination and axonal loss in multiple sclerosis: a comparison with conventional $\mathrm{T}_{2}$-weighted images. F Neurol 1997;244: 266-70.

25 Studholme C, Hill DLG, Hawkes DJ. Automated 3D registration of MR and PET brain images by multi-resolution optimisation of voxel similarity measures. Med Physics 1997;24:25-35.

26 Filippi M, Bozzali M, Horsfield MA, et al. A conventional and magnetization transfer MRI study of the cervical cord in patients with MS. Neurology 2000;54:207-13.

27 van Buchem MA, McGowan JC, Kolson DL, et al. Quantitative volumetric magnetization transfer analysis in multiple sclerosis: estimation of macroscopic and microscopic ple sclerosis: estimation of macroscopic and micre burden. Magn Reson Med 1996;36:632-6.

28 Cercignani M, Iannucci G, Rocca MA, et al. Pathological damage in MS assessed by diffusion-weighted and magnetization transfer MRI. Neurology 2000;54:1139-44.

29 Adams JH, Blackwood W, Wilson J. Further clinical and pathological observations on Leber's optic atrophy. Brain 1966;89:15-26.

30 van Waesberghe JH, Kamphorst W, DeGroot CJ, et al. Axonal loss in multiple sclerosis lesions: magnetic resonance imaging insights into substrates of disability. Ann Neurol 1999;46:747-54.

31 Thorpe JW, Barker GJ, Jones SJ, et al. Magnetisation transfer ratios and transverse magnetisation decay curves in optic neuritis: correlation with clinical findings and electrophysiology. F Neurol Neurosurg Psychiatry 1995;59:487-92.

32 Boorstein JM, Moonis G, Boorstein SM, et al. Optic neuritis: imaging with magnetisation transfer. $A \mathcal{F R} A m \mathcal{F}$ Roentgenol 1997;169:1709-12.

33 Filippi M, Yousry TA, Baratti C, et al. Quantitative assessment of MRI lesion load in multiple sclerosis: a comparison of conventional spin-echo with fast fluidattenuated inversion recovery. Brain 1996;119:1349-55.

34 Gawne-Cain ML, O'Riordan JI, Thompson AJ, et al. Multiple sclerosis lesion detection in the brain: a comparison of fast fluid-attenuated inversion recovery and conventional T2-weighted dual spin-echo. Neurology 1997;49:364-70.

35 Ferini-Strambi L, Bozzali M, Cercignani M, et al. Magnetization transfer and diffusion-weighted imaging in nocturnal frontal lobe epilepsy. Neurology 2000;54:2331-3.

36 Tartaglino LM, Friedman DP, Flanders AE, et al. Multiple sclerosis in the spinal cord: MR appearance and correlation with clinical parameters. Radiology 1995;195:725-32.

37 Campi A, Filippi M, Comi G, et al. Acute transverse myelopathy: spinal and cranial MR study with clinical follow-up. AfNR Am 7 Neuroradiol 1995;16:115-23. 